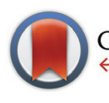

CrossMark

Cite this: Dalton Trans., 2016, 45 18921

Received 10th October 2016, Accepted 8th November 2016 DOI: $10.1039 / c 6 d t 03906 e$ www.rsc.org/dalton

\section{Mechanistic insights into excited state intramolecular proton transfer in isolated and metal chelated supramolecular chemosensors $\uparrow$}

\author{
Tolga N. V. Karsili, ${ }^{a, b}$ Barbara Marchetti $^{\mathrm{a}, \mathrm{b}}$ and Michael N. R. Ashfold ${ }^{\mathrm{b}}$
}

We report a computational study of excited state intramolecular proton transfer in a series of related and progressively more complex organic chromophores ranging from 2-(2'-hydroxyphenyl)-benzoxazole (HBO) through to the 5-(benzo[d]oxazol-2-yl)-2-(4-((bis(pyridin-2-ylmethyl)amino)methyl)benzo[d] oxazol-2-yl)-4 hydroxyphenolate (HDBO') anion. The latter chelates group 12 metal cations $\left(X=\mathrm{Zn}^{2+}\right.$, $\mathrm{Cd}^{2+}$ and $\mathrm{Hg}^{2+}$ ), and can serve as a fluorescence-based sensor for such metals. Initial $\pi^{\star} \leftarrow \pi$ excitation of the ground $\left(\mathrm{S}_{0}\right)$ state enol-tautomer induces charge separation in the first excited singlet $\left(\mathrm{S}_{1}\right)$ state and drives the subsequent proton transfer (i.e. enol $\rightarrow$ keto tautomerism). The keto-tautomer constitutes a local minimum on the $S_{1}$ PES, and is responsible for highly Stokes shifted fluorescent emission; $S_{1}(e n o l) \rightarrow$ $\mathrm{S}_{0}$ fluorescence is proposed to account for the shorter wavelength emission from the $\mathrm{X}-\mathrm{HDBO}$ ' complexes. Derivatives of HDBO' that should retain the favourable visible absorption and heavily Stokes shifted emission properties but, additionally, offer higher fluorescence quantum yields (i.e. enhanced metal sensing capability) are proposed.

\section{Introduction}

Luminescent sensors are typically functionalised supramolecules that enhance or quench fluorescence (or phosphorescence) upon an external stimulus, such as a change in the chemical environment. ${ }^{1,2}$ Such species are generally optimised synthetically in order to maximise their selectivities and specificities whilst steering their photophysical properties towards a particular intended function. Many studies have focussed on the synthesis of luminescent supramolecules that are specific for the detection of malignant tumours. ${ }^{3}$ Upon detection and subsequent complexation to specific markers released by cancerous tissue, changes to the luminescence signal and the Stokes shift reveal their specific positions and activities, thus enhancing the efficiency and efficacy of the diagnosis. Hence the major interest in photochemically active supramolecules as bio-diagnostics. $^{4,5}$

\footnotetext{
${ }^{a}$ Department of Chemistry, Technische Universität München, Lichtenbergstrasse 4, 85748 Garching, Germany. E-mail: tolga.karsili@tum.de

${ }^{b}$ School of Chemistry, University of Bristol, Cantock's Close, Bristol, BS8 1TS, UK $\dagger$ Electronic supplementary information (ESI) available: (i) Minimum energy geometries and dominant orbital promotions associated with excitation to the lowest three singlet excited states of $\mathrm{Cd}^{2+}-\mathrm{HDBO}^{\prime}$ and $\mathrm{Hg}^{2+}-\mathrm{HDBO}^{\prime}$; (ii) permanent dipole moment vectors in the $\mathrm{S}_{0}$ and $\mathrm{S}_{1}$ (enol and keto) states of HBO. See DOI: $10.1039 / \mathrm{c} 6 \mathrm{dt} 03906 \mathrm{e}$
}

Trace metal detection in, for example, water supply systems is another crucial analytical problem and one that is required for the regulation of safe drinking water. ${ }^{6}$ Supramolecular systems that are benign towards human health are ideal for this purpose, allowing their addition to the water supply to test and ultimately regulate the concentrations of trace metals. Binding of such metals to the supramolecule causes a measurable (photo)chemical change. One such example is the detection of alkali metal cations using N-bridgehead cryptandnaphthalene supramolecules which, when cation-bound, fluoresce following electronic excitation. ${ }^{7}$ In the absence of the metal, however, photoexcitation of the free supramolecule causes photo-induced electron transfer (PET) from the naphthalene donor to the cryptand-hole acceptor; the fluorescence quantum yield is negligible. The mechanisms responsible for this switch in behaviour are, as yet, poorly understood.

Other studies have demonstrated the tunability of such supramolecular sensors. ${ }^{8-10}$ Aza-crown ethers, for example, with chromophores (such as coumarin) attached to both ends, have been shown to exhibit similar, but opposite, PET and fluorescence propensities, i.e. the supramolecule fluoresces when metal-free but undergoes PET when metal-bound. ${ }^{11}$ These systems are also referred to as molecular LOGIC switches, since they 'switch' the fluorescence or PET signal 'on' or 'off' depending on the presence or absence of the 
intended 'to be detected' solute. ${ }^{12}$ In these specific examples, sensing relies on a change in the electronic properties of the sensor upon chelation with the alkali metal. Such donoracceptor supramolecules can also be tuned, synthetically, in order to function as molecular nanowires. ${ }^{13-16}$

As well as changes in electronic structure, molecules used as luminescent probes can also undergo geometry changes upon chelation and/or (subsequent) photoexcitation. One notable example is $E$-diazobenzene, which can function as a molecular photo-switch upon irradiation with light and has found application in $\mathrm{pH}$ sensing, photochromism and in nonlinear optics. ${ }^{17}$ These applications all rely on the same photophysics: upon near-UV irradiation, $E$-diazobenzene undergoes $E \rightarrow Z$ photoisomerism on the excited state potential energy surface (PES), followed by rapid internal conversion (IC) and subsequent branching into either $Z$ - $(30 \%)$ or $E$-diazobenzene (70\%) on the ground state PES. The timescales of such $E \rightarrow Z$ photoisomerisms and subsequent IC processes are typically ultrafast - sub-ps in the specific case of diazobenzene, as revealed by transient absorption studies in deuterated dimethyl sulfoxide ${ }^{18}$ and $n$-hexane solutions. ${ }^{19}$ Such ultrafast IC is generally associated with the presence of low energy crossings between adiabatic PESs which, when orthogonal motions are considered, develop into conical intersections (CIs) ${ }^{20}$ CIs are ubiquitous in photochemically and excited state driven phenomena and are recognised as regions of the PES that promote efficient transfer of excited state population back to the ground state (or between excited states). Such processes are termed non-adiabatic, as the electronic and nuclear motions are usually strongly coupled.

Non-adiabatic processes can be driven by a variety of nuclear motions, but the present study focusses on proton/ electron coupled motions - commonly referred to as protoncoupled electron transfer (PCET) processes - which drive much of the redox chemistry associated with transition metal complexes. ${ }^{21}$ In a hydrogen-bonded complex, photoinducedPCET occurs when light absorption initiates electron promotion from an acidic donor (D) orbital to a hitherto unoccupied, basic acceptor (A) orbital. This creates a $\mathrm{D}^{+} \mathrm{H}-\mathrm{A}^{-}$chargeseparated (CS) excited state that is neutralised by transfer of a proton from D to A. The potential energy of a CS excited state almost always decreases upon proton transfer (PT), i.e. there is a driving force for PT in a CS excited state. The ground state, in contrast, is stable in the closed-shell DH-A configuration, so its PES shows a large barrier with respect to PT. Thus the CS and ground state potentials inevitably cross along the PT coordinate and the resulting $\mathrm{CI}$ facilitates non-adiabatic decay of CS state population to the ground state - i.e. facilitates ultrafast PCET. In cases where the PT occurs at short range, the charge separation in the excited CS state may not fully neutralise and further nuclear rearrangements may be necessary to cancel the remaining charge-separation ultimately leading to a curve crossing. ${ }^{22-25}$ Such molecular distortions may be restricted in strained molecular systems, however. In these cases, the excited state population may be trapped in a metastable CS state, which then decays to the ground state by an alternative route, by interacting with surrounding solvent molecules or, in extreme cases, by fluorescing. The fluorescence from such constrained systems can be exploited when designing effective supramolecular fluorescent probes $^{22,23,26}$ - as we illustrate here. The class of (supra-)molecular sensors known to function via PCET is still rather small but, as shown in a recent review, ${ }^{27}$ is growing fast.

The present study explores the mechanism of excited state intramolecular proton-transfer (ESIPT) (a specific class of PCET) in a series of organic chromophores that can be tuned synthetically so as to function as supramolecular chemosensors for trace metal detection. We start with 2-(2'-hydroxyphenyl)-benzoxazole (HBO, depicted in Fig. 1), which is known to undergo ESIPT upon electronic excitation. ${ }^{27}$ Transient absorption measurements following $340 \mathrm{~nm}$ excitation of HBO in cyclohexane solution show enol $\rightarrow$ keto tautomerism occurring as a result of ESIPT on a timescale of $\sim 100$ fs or less ${ }^{28-31}$ - consistent with the results of mixed quantum/classical molecular dynamics calculations by Daengngern et $a l^{32}$ The thio-analogue of $\mathrm{HBO}$ has also been studied recently by Barbatti and co-workers ${ }^{33}$ who proposed, on the basis of the deduced ultrafast excited state intra- and inter-molecular PT, that this molecule could be a good candidate for use as a protein fluorescent marker.

The potential utility of the fast and efficient ESIPT in HBO is likely to be limited by its absorption maximum $\left(\lambda_{\mathrm{abs}(\max )}\right.$ $\sim 330 \mathrm{~nm}$ ), however, which is much shorter than the (visible) wavelengths preferred for supramolecular sensing applications. ${ }^{34}$ Adding another benzoxazole to $\mathrm{HBO}$ yields $2-\left(2^{\prime}\right.$ hydroxyphenyl)-dibenzoxazole (HDBO, termed bis(HBO) in ref. 34, and also shown in Fig. 1), shifts the absorption maximum closer to the visible $\left(\lambda_{\mathrm{abs}(\max )}=412 \mathrm{~nm}\right)$ and still maintains the ESIPT upon photoexcitation. ${ }^{34}$ Functionalising HDBO with $\mathrm{N}, \mathrm{N}$-dipyridylmethylamine results in formation of 5 -(benzo[d] oxazol-2-yl)-2-(4-((bis( pyridin-2-ylmethyl)amino)methyl)benzo [d]oxazol-2-yl)-4 hydroxyphenolate (henceforth termed HDBO', but abbreviated as Zinhbo-1 in ref. 34). Protonated HDBO' shows a similar absorption maximum $\lambda_{\text {abs(max) }} \sim 410 \mathrm{~nm}$ in non-polar solvents, attributable to the neutral free ligand, and a weak fluorescent emission centred at $\lambda_{\mathrm{em}}=543 \mathrm{~nm}$ (i.e. a Stokes shift of $\sim 130 \mathrm{~nm})$. HDBO' is of particular interest as a ligand sensor for trace metal detection in more polar solvents. The group $12 \mathrm{X}-\mathrm{HDBO}^{\prime}$ complexes $\left(\mathrm{X}=\mathrm{Zn}^{2+}, \mathrm{Cd}^{2+}\right.$ and $\mathrm{Hg}^{2+}$, with the phenol proximal to the cation deprotonated, also illustrated in Fig. 1) show much larger Stokes shifts than bare HDBO'. $\mathrm{Zn}^{2+}-\mathrm{HDBO}^{\prime}$, for example, shows $\lambda_{\text {abs(max) }} \sim 480 \mathrm{~nm}$ both in DMSO and in aqueous solution, and $\lambda_{\mathrm{em}}=712 \mathrm{~nm}-\mathrm{a}$ spectacularly large Stokes shift of $\sim 230 \mathrm{~nm} .^{34}$

The present work reports gas phase (isolated molecule) electronic structure calculations designed to offer mechanistic insights into these photoinduced turn-on processes. Specifically, we explore the topographies of the excited state PESs that support these efficient ESIPT processes, the origin of the large differences in Stokes shift upon progressing from $\mathrm{HBO}$ to $\mathrm{HDBO}$ to $\mathrm{X}-\mathrm{HDBO}^{\prime}$, and the ways in which chelation with metal ions affects the potential energy (PE) profile along 

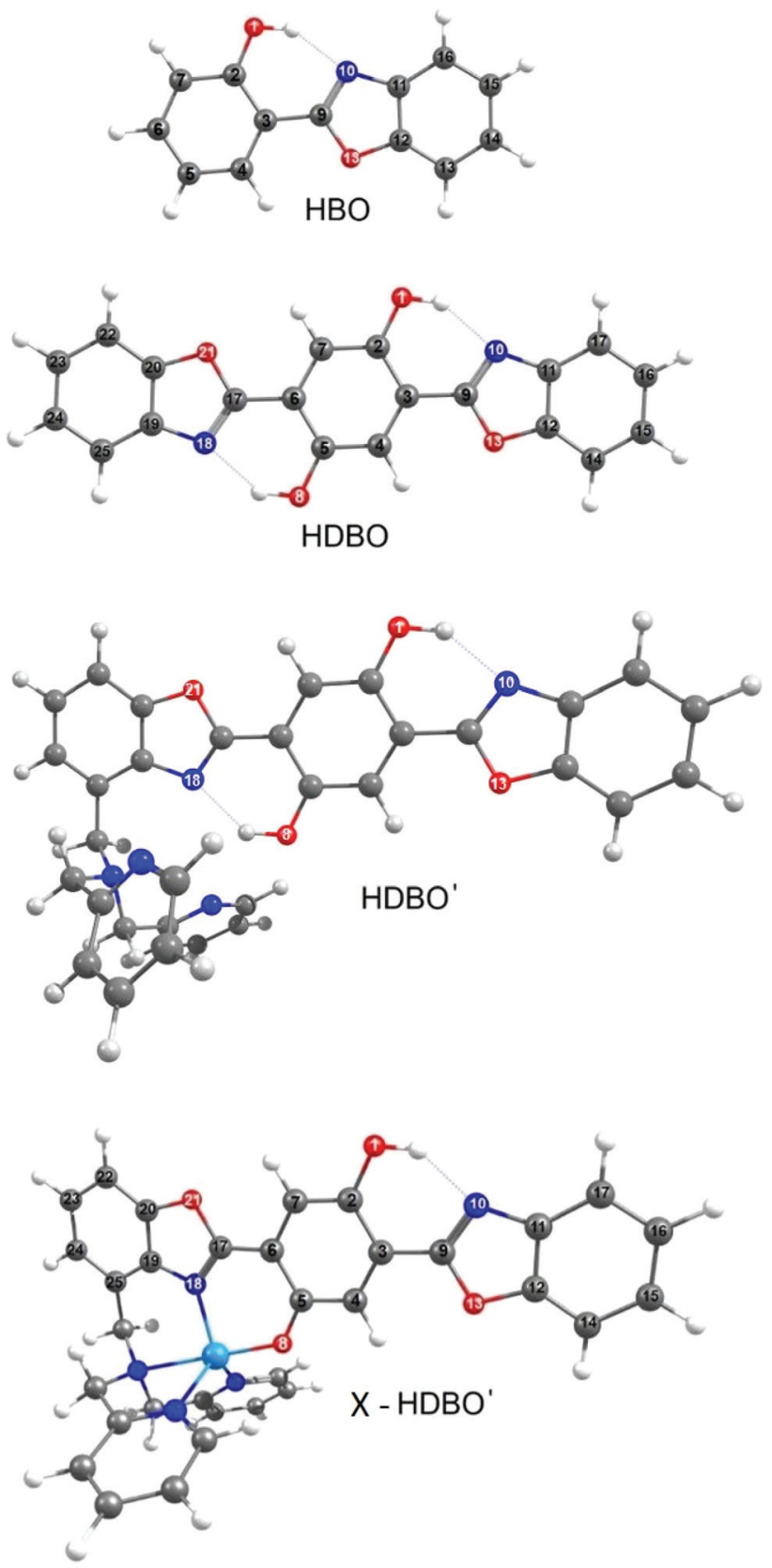

Fig. 1 Depiction of the minimum energy geometries of HBO, HDBO, protonated HDBO' and X-HDBO' (where $\mathrm{X}=\mathrm{Zn}^{2+}, \mathrm{Cd}^{2+}$ or $\mathrm{Hg}^{2+}$ and the HDBO' ligand is singly deprotonated) optimized at the DFT/CAM-B3LYP/ $6-31 \mathrm{G}(\mathrm{d})$ level of theory. The dotted lines between $\mathrm{O}(1) \mathrm{H} \cdots \mathrm{N}(10)$ and, in all but $\mathrm{HBO}$, between $\mathrm{O}(8) \mathrm{H} \cdots \mathrm{N}(18)$ indicate intramolecular hydrogen bonds and the numbers assigned to the various heavy atoms are required for orientation purposes later.

the ESIPT coordinate and the Stokes shift. In so doing, we also suggest ways in which it may be possible to enhance the fluorescence quantum yield (i.e. the sensor sensitivity) by further chemical modification of the $\mathrm{HBDO}^{\prime}$ ligand in an effort to reduce the efficiency of competing non-radiative excited state decay pathways.

\section{Computational methodology}

Using the Gaussian $09^{35}$ computational package, the ground state minimum energy geometries of all species of current interest were optimized using the long-range correlated Coulomb Attenuated Model Becke- ${ }^{\text {rd }}$ parameter-Lee-YangParr (CAM-B3LYP) functional ${ }^{36}$ in Density Functional Theory (DFT).

Relaxed PE profiles for the ground and first few excited electronic states of all systems investigated were scanned along the ESIPT coordinate using time dependent (TD)-DFT/CAM-B3LYP using the $\mathrm{O} 1-\mathrm{H}$ bond length $R_{\mathrm{O} 1-\mathrm{H}}$, as the driving coordinate. A 6-31G(d) ${ }^{37}$ Pople basis set was used for all first and second row atoms (i.e. $\mathrm{H}, \mathrm{C}, \mathrm{N}$ and $\mathrm{O}$ ), together with 6-31G(d) (for $\mathrm{Zn}$ ) and $\mathrm{LANL}_{2} \mathrm{DZ}^{38}$ (for $\mathrm{Cd}$ and $\mathrm{Hg}$ ) basis functions for calculations of the metal complexes.

$\mathrm{S}_{1}-\mathrm{S}_{0}$ absorption profiles of $\mathrm{HBO}$ and $\mathrm{HDBO}$ were simulated using Newton-X, ${ }^{39,40}$ by first generating an ensemble of 100 initial geometries $(N)$ - sampled using a Wigner distribution based on the ground state normal mode harmonic wavenumbers (calculated using the system specific level of theory described above). Following ref. 41, the vertical excitation energy and oscillator strength were then calculated for each of the $N$ initial geometries using TD-DFT/CAM-B3LYP/6-31G(d) and the excitation energy dependent photoabsorption cross section $P(E)$ obtained using eq. (1)

$$
P(E)=\frac{\pi e^{2}}{2 m c \varepsilon_{0}} \sum_{i=0}^{j=1-3}\left[\frac{1}{N_{\text {tot }}} \sum_{N=1}^{100} f_{i j}\left(R_{k}\right) g\left(E-\Delta E\left(R_{k}\right), \delta\right)\right] .
$$

The internal sum in eqn (1) is over the set of Wigner points $\left(N_{\text {tot }}=100\right)$ while the external sum includes transitions from the initial state ( $i$, the $\mathrm{S}_{0}$ state) to final states $\left(j=\mathrm{S}_{1}, \mathrm{~S}_{2}\right.$ and $\mathrm{S}_{3}$ in the present modelling) with respective oscillator strengths $f_{i j}$. $R_{k}$ represents the initial coordinates of the $N^{\text {th }}$ point and $g(E-$ $\left.\Delta E\left(R_{k}\right), \delta\right)$ is a Lorentzian line shape function given by eqn (2)

$$
g\left(E-\Delta E\left(R_{k}\right), \delta\right)=\frac{\hbar \delta}{2 \pi}\left(\left(E-\Delta E_{i j}\right)^{2}+\left(\frac{\delta}{2}\right)^{2}\right)^{-1} .
$$

$\delta$ in eqn (2) is a broadening factor, which was arbitrarily set to $0.05 \mathrm{eV}$ for each of the calculated absorption profiles reported here. $\mathrm{S}_{1}-\mathrm{S}_{0}$ emission profiles were calculated in a similar manner, by sampling an ensemble of 100 initial geometries using a Wigner distribution based on the normal mode wavenumbers associated with the optimized geometry of the first singlet excited electronic state (calculated using the CAM-B3LYP/6-31G(d) level of theory as above).

\section{Results and discussion}

\subsection{Ground state minimum energy geometry and vertical excitation energies}

Fig. 1 showed the ground state minimum energy geometries of $\mathrm{HBO}, \mathrm{HDBO}$, protonated $\mathrm{HDBO}^{\prime}$ and $\mathrm{Zn}^{2+}-\mathrm{HDBO}^{\prime}$. The $\mathrm{Cd}^{2+}-$ $\mathrm{HDBO}^{\prime}$ and $\mathrm{Hg}^{2+}-\mathrm{HDBO}^{\prime}$ complexes display the same struc- 
Table 1 Vertical excitation energies (in eV, defined relative to $E\left(\mathrm{~S}_{0}\right)=0$ ) to the first three singlet and triplet (i.e. $j=1-3$ ) excited states of $\mathrm{HBO}$, HDBO, protonated and deprotonated HDBO' and the three X-HDBO' complexes of current interest, calculated using (TD)-DFT/CAM-B3LYP, along with calculated $\mathrm{S}_{j}-\mathrm{S}_{0}$ oscillator strengths

\begin{tabular}{|c|c|c|c|}
\hline \multirow[b]{2}{*}{$i$} & \multicolumn{2}{|c|}{ Vertical excitation energy/eV } & \multirow{2}{*}{$\begin{array}{l}\mathrm{S}_{J}-\mathrm{S}_{0} \text { oscillator } \\
\text { strength, } f\end{array}$} \\
\hline & $\mathrm{S}_{i}$ & $\mathrm{~T}_{i}$ & \\
\hline \multicolumn{4}{|c|}{$\mathrm{HBO}$} \\
\hline 1 & 4.34 & 2.96 & 0.447 \\
\hline 2 & 4.97 & 3.62 & 0.261 \\
\hline 3 & 5.37 & 3.63 & 0.063 \\
\hline \multicolumn{4}{|c|}{ HDBO } \\
\hline 1 & 3.40 & 2.31 & 0.538 \\
\hline 2 & 4.36 & 2.86 & 0.988 \\
\hline 3 & 4.95 & 3.32 & 0.000 \\
\hline \multicolumn{4}{|c|}{ Protonated HDBO' } \\
\hline 1 & 3.40 & 2.31 & 0.508 \\
\hline 2 & 4.34 & 2.87 & 0.921 \\
\hline 3 & 4.76 & 3.29 & 0.012 \\
\hline \multicolumn{4}{|c|}{ Deprotonated HDBO' } \\
\hline 1 & 2.22 & 1.02 & 0.251 \\
\hline 2 & 3.11 & 2.75 & 0.000 \\
\hline 3 & 3.58 & 2.95 & 0.166 \\
\hline \multicolumn{4}{|c|}{$\mathrm{Zn}^{2+}-\mathrm{HDBO}^{\prime}$} \\
\hline 1 & 2.96 & 1.92 & 0.335 \\
\hline 2 & 3.75 & 2.70 & 0.001 \\
\hline 3 & 3.81 & 3.30 & 0.000 \\
\hline \multicolumn{4}{|c|}{$\mathrm{Cd}^{2+}-\mathrm{HDBO}^{\prime}$} \\
\hline 1 & 2.82 & 1.83 & 0.307 \\
\hline 2 & 3.56 & 2.61 & 0.002 \\
\hline \multirow{2}{*}{\multicolumn{4}{|c|}{$\mathrm{Hg}^{2+}-\mathrm{HDBO}^{\prime}$}} \\
\hline & & & \\
\hline 1 & 2.81 & 1.84 & 0.286 \\
\hline 2 & 3.59 & 2.61 & 0.002 \\
\hline 3 & 3.66 & 3.21 & 0.000 \\
\hline
\end{tabular}

tural arrangement as $\mathrm{Zn}^{2+}-\mathrm{HDBO}^{\prime}$ and are thus only shown in Fig. S1 of the ESI. $\dagger$ The minimum energy structure in each case involves planar $\mathrm{HBO}$ and $\mathrm{HDBO}$ moieties. The acidic $\mathrm{O}(1)-\mathrm{H}$ group shows an intramolecular hydrogen bond with the basic $\mathrm{N}(10)$ atom in all species, as does the $\mathrm{O}(8)-\mathrm{H}$ group (with the $\mathrm{N}(18)$ atom) in $\mathrm{HDBO}$ and protonated $\mathrm{HDBO}^{\prime}$; both of these $\mathrm{N}$ atoms possess an in-plane lone pair that can act as the hydrogen bond acceptor.

Table 1 lists vertical excitation energies (VEEs) and oscillator strengths for the $\mathrm{S}_{j} \leftarrow \mathrm{S}_{0}(j=1-3)$ transitions returned by the TD-DFT calculations for all systems featured in the present study, while the dominant associated orbital promotions are shown in Fig. 2. For completeness, the calculated VEEs to the corresponding triplet excited states are also included in Table 1; the PE profiles of some of the latter states will be considered later, when considering the metal complexes (vide infra).

The first three singlet excited states of HBO and HDBO are each calculated to be of $\pi \pi^{*}$ character. In both cases, the excitation from the $\pi$ highest occupied molecular orbital (HOMO) to the lowest unoccupied $\pi^{*}$ (LUMO) as shown in Fig. 2 is calculated to contribute $>80 \%$ of the total weight of the $S_{1}-S_{0}$ transition. These transitions have appreciable oscillator strengths, and their respective VEEs are in reasonable accord with the long wavelength absorption maxima determined by experiment. The calculated $\mathrm{S}_{1}-\mathrm{S}_{0}$ VEE in HBO (Table 1) corresponds to a wavelength $\lambda_{\text {abs, calc }} \sim 285 \mathrm{~nm}$ ( $c f$. the experimental $\lambda_{\text {abs(max) }} \sim 330 \mathrm{~nm}$ ), while the equivalent comparison in the case of HDBO is $\lambda_{\text {abs, calc }} \sim 365 \mathrm{~nm}, c f$. $\lambda_{\text {abs(max) }} \sim 412 \mathrm{~nm} .{ }^{34}$ We should expect this (already acceptable) level of agreement
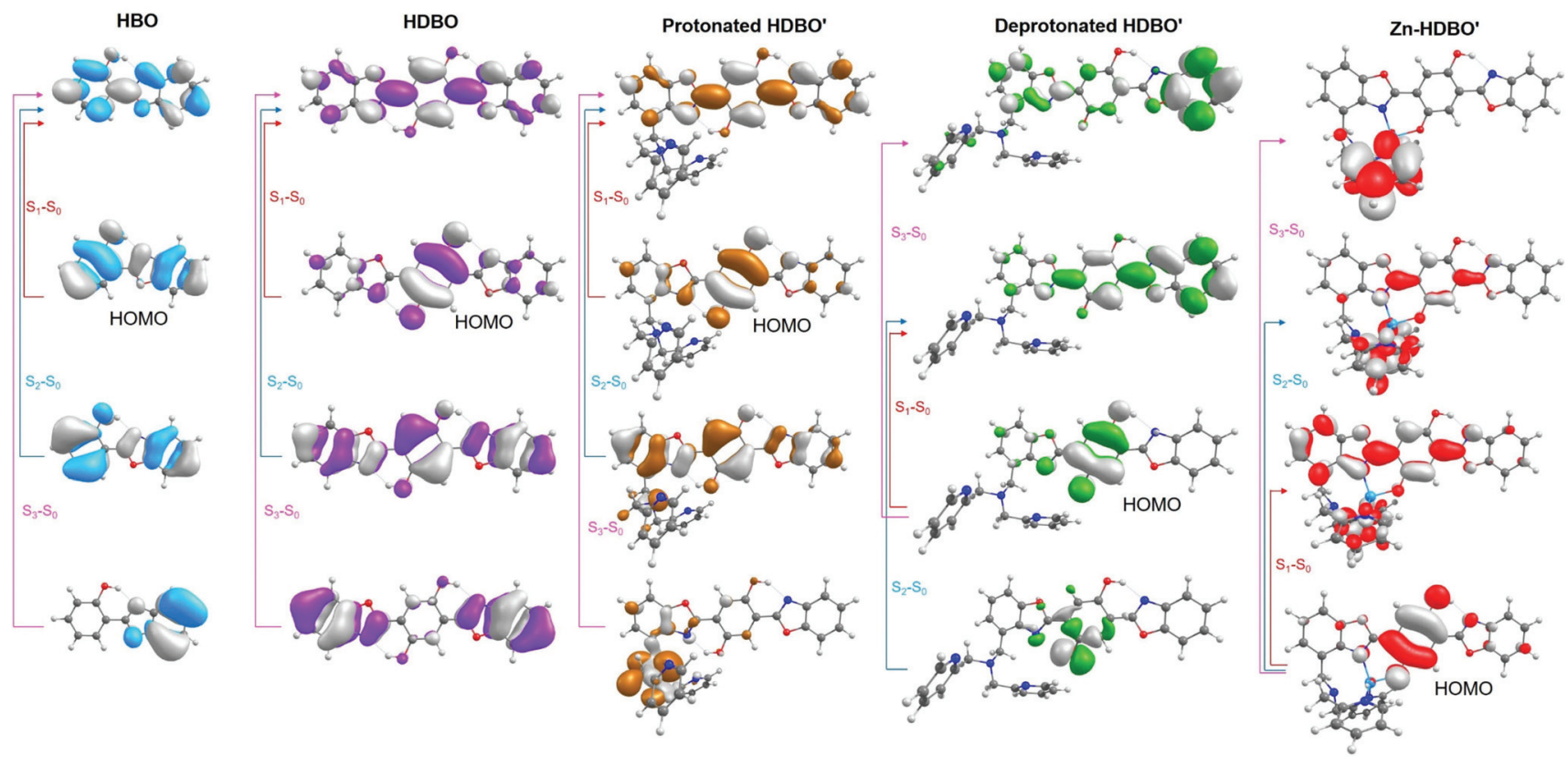

Fig. 2 Depictions of the orbitals involved in the dominant electron promotions responsible for the first three singlet excited electronic states of $\mathrm{HBO}, \mathrm{HDBO}$, protonated and deprotonated $\mathrm{HDBO}^{\prime}$, and the $\mathrm{Zn}^{2+}-\mathrm{HDBO}^{\prime}$ complex. The HOMO is indicated in each case. 
between theory and experiment to improve further once zeropoint energy differences are included. The $S_{2}-S_{0}$ and $S_{3}-S_{0}$ transitions are only poorly described in terms of single orbital promotions; in both molecules, the orbital promotions shown in Fig. 2 are calculated to be dominant, but only to contribute $\sim 40 \%$ of the total transition strength.

The electronic promotions responsible for the $\mathrm{S}_{1}$ and $\mathrm{S}_{2}$ states of protonated $\mathrm{HDBO}^{\prime}$ are analogous to those for HDBO, whereas the largest contributor to the $S_{3}$ state is predicted to involve electron promotion from an orbital centred on the $N, N$-dipyridylmethylamine group to the analogous $\pi^{*}$ LUMO as in HDBO. Theory (Table 1) and experiment both suggest that functionalization with $N, N$-dipyridylmethylamine has little impact on the long wavelength $S_{1}-S_{0}$ absorption of HDBO.

Upon deprotonating $\mathrm{O}(8)$, this and the $\mathrm{N}(18)$ atom are now both effective donor sites enabling complexation to metal atoms with low oxidation states. The same orbital promotions dominate when forming the $\mathrm{S}_{1}$ states of protonated and deprotonated $\mathrm{HDBO}^{\prime}$ but, as Table 1 shows, the calculated VEE of the $S_{1}-S_{0}$ transition in the latter is significantly reduced. This can be rationalised in light of the loss of conjugation upon deprotonating $\mathrm{O}(8)$. As Fig. 2 shows, deprotonating $\mathrm{HDBO}^{\prime}$ destabilises the HOMO by increasing the $\pi$-density on $\mathrm{O}(8)$ and thus reducing the conjugation of the $\mathrm{C}(5)=\mathrm{O}(18)$ bond. The $\pi^{*}$ LUMO, in contrast, is largely unaffected. Thus the net effect of deprotonating $\mathrm{O}(8)$ is a reduction in the HOMO-LUMO energy gap - consistent with the bathochromic shift of the $\mathrm{S}_{1}-\mathrm{S}_{0}$ absorption maximum observed for the singly deprotonated, unchelated $\mathrm{HDBO}^{\prime}$ moiety in aqueous solution. $^{34}$

The visible absorption maximum shifts further upon chelation with $\mathrm{Zn}^{2+}, \mathrm{Cd}^{2+}$ or $\mathrm{Hg}^{2+}$. The metal atoms in these complexes all have filled d shells so, as Fig. 2 shows, the HOMO in each case is similar to that in HDBO and the first excited states are formed by ligand centred $\pi^{*} \leftarrow \pi$ transitions. As Table 1 shows, the respective $S_{1}-S_{0}$ transitions are each predicted to have substantial oscillator strengths and to show a bathochromic shift relative to the corresponding transitions in HBO, HDBO and protonated $\mathrm{HDBO}^{\prime}$ - again in accord with experimental observation ${ }^{34}$ - but to be blue-shifted relative to that in deprotonated $\mathrm{HDBO}^{\prime}$. The latter trend is understandable in terms of a partial recovery of conjugation within the ligand $\pi$-system induced by dative bonding to the metal centre. We recognise that heavy metal species like $\mathrm{Hg}^{2+}$, in particular, are likely to induce strong spin-orbit coupling, but the inclusion of such effects is beyond the scope of the present work. The $\mathrm{S}_{2}-\mathrm{S}_{0}$ and $\mathrm{S}_{3}-\mathrm{S}_{0}$ transitions in these X-HDBO' complexes also involve $\pi^{*} \leftarrow \pi$ excitations. Reference to Fig. 2 and $\mathrm{S} 1 \dagger$ suggests that the energetic ordering of these closely spaced $\pi^{*}$ orbitals is $\mathrm{X}$ dependent, but in all cases, one of these $\pi^{*}$ orbitals is largely localised on the $N, N$-dipyridylmethylamine moiety while the other is delocalised over the entire HDBO' ligand. Neither overlap well with the $\pi$ HOMO, thus accounting for the small oscillator strengths calculated for these transitions (Table 1).

\subsection{Excited state intramolecular proton transfer, emission and Stokes shift}

HBO and HDBO. Fig. 3(a) and (b) show PE profiles for the ground and first few excited states of, respectively, $\mathrm{HBO}$ and HDBO along the ESIPT coordinate. In both cases, the filled black circles indicate the relaxed profile of the ground state along $R_{\mathrm{O}(1)-\mathrm{H}}$ and the open coloured circles show the energies of various singlet and triplet excited states calculated at the corresponding $\mathrm{S}_{0}$ relaxed geometries along $R_{\mathrm{O}(1)-\mathrm{H}}$. The profile shown by the filled red circles is for the relaxed geometries of the $\mathrm{S}_{1}$ state along $R_{\mathrm{O}(1)-\mathrm{H}}$, while the open black circles show the $S_{0}$ energies at the corresponding relaxed $S_{1}$ geometries.

Fig. 3 confirms that the enol tautomer is the minimum energy structure in the electronic ground states of both HBO and HDBO and that the keto tautomer corresponds to a local
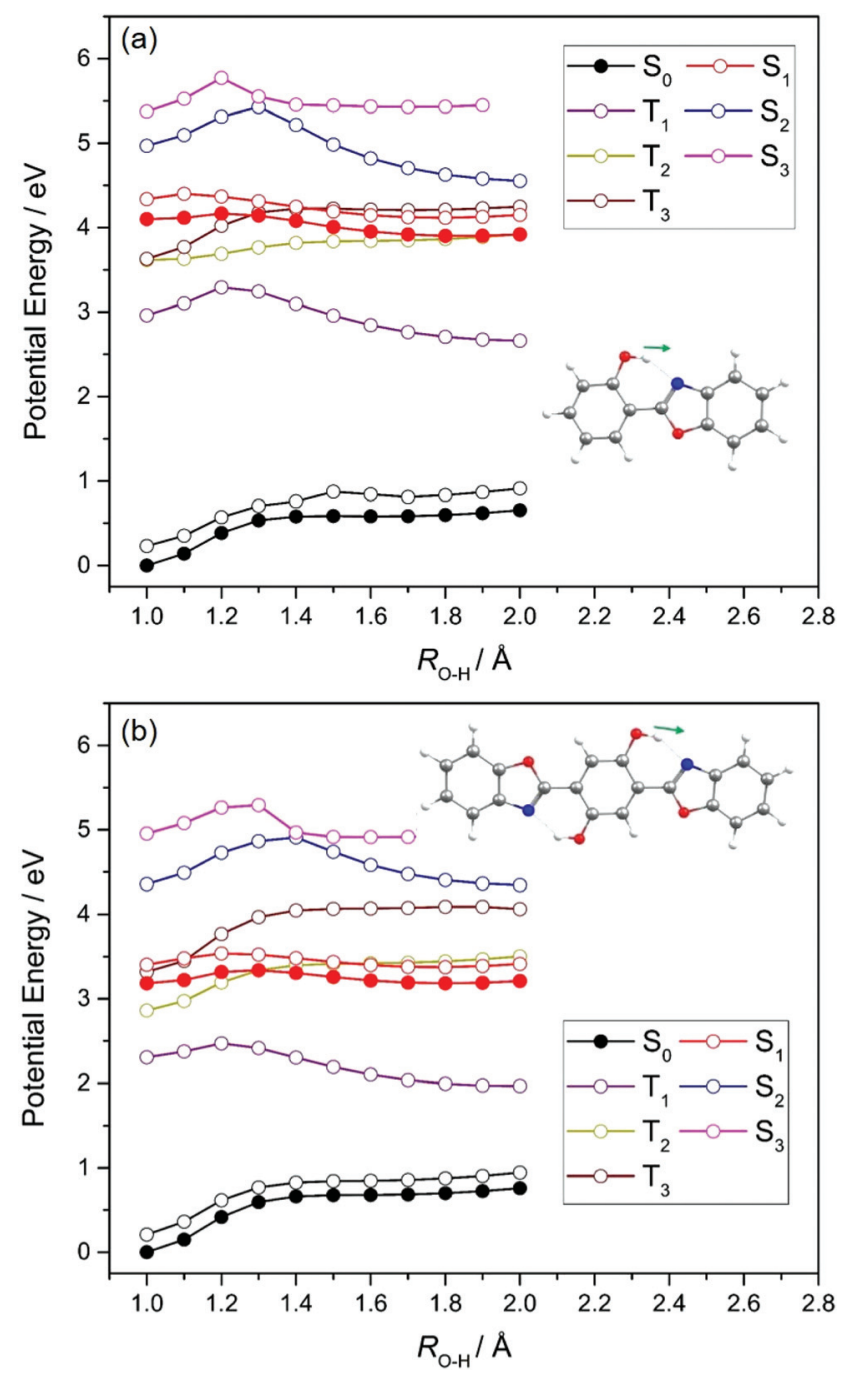

Fig. 3 PE profiles along the $R_{\mathrm{O}(1)-\mathrm{H}}$ ESIPT coordinate in (a) $\mathrm{HBO}$ and (b) HDBO. The black and red filled circles show the relaxed profiles for, respectively, the $S_{0}$ and $S_{1}\left(1^{1} \pi \pi^{\star}(C T)\right)$ states, while the open black circles show the energy of the $S_{0}$ state computed at the relaxed $S_{1}$ state geometries. Other PE profiles shown by colored open circles are for the excited states indicated, calculated at the relaxed $\mathrm{S}_{0}$ state geometries. 
maximum along $R_{\mathrm{O}(1)-\mathrm{H}}$. The respective $\mathrm{S}_{1}$ potentials, in contrast, show minima for both enol and keto tautomers, with the latter calculated to be more stable by $\sim 0.3 \mathrm{eV}$ in $\mathrm{HBO}$, and by $\sim 0.1 \mathrm{eV}$ in the case of HDBO. The calculated barrier to enol $\rightarrow$ keto tautomerism on the $\mathrm{S}_{1}$ PES of $\mathrm{HBO}$ is only $\sim 0.05 \mathrm{eV}$; the corresponding barrier for $\mathrm{HDBO}$ is calculated to be larger $(\sim 0.13 \mathrm{eV})$. Thus the present calculations reveal a clear driving force for enol $\rightarrow$ keto tautomerism following vertical excitation to the $S_{1}$ state of $\mathrm{HBO}$. This accords with the predicted change in both the magnitude and direction of the permanent dipole moment upon electronic excitation (see Fig. S2 of ESI $\dagger$ ), and indicates that the $S_{1}$ state develops substantial CT character upon extending $R_{\mathrm{O}(1)-\mathrm{H}}$. Based solely on the present calculations, the tautomerism probability following vertical excitation in HDBO is less clear cut and further complicated by the additional $\mathrm{O}(8) \mathrm{H} \cdots \mathrm{N}(18)$ hydrogen bond, which opens the possibility of double ESIPT (along the $R_{\mathrm{O}(1)-\mathrm{H}}$ and $R_{\mathrm{O}(8)-\mathrm{H}}$ coordinates).

Fig. 4 shows calculated $S_{0}$ and $S_{1}$ PESs of HDBO plotted as functions of $R_{\mathrm{O}(1)-\mathrm{H}}$ and $R_{\mathrm{O}(8)-\mathrm{H}}$. For each $R_{\mathrm{O}(1)-\mathrm{H}}$ and $R_{\mathrm{O}(8)-\mathrm{H}}$ combination, the $S_{0}$ energy was calculated after relaxing all other nuclear degrees of freedom and the $S_{1}$ energies were then computed at these relaxed $S_{0}$ geometries. The $S_{1}$ PES calculated in this way displays minima corresponding to the keto tautomers formed by single proton transfer along either $R_{\mathrm{O}(1)-\mathrm{H}}$ and $R_{\mathrm{O}(8)-\mathrm{H}}$. These are equivalent, as required by the parent symmetry. The $\mathrm{S}_{1}$ PES also shows a local minimum at the geometry corresponding to double ESIPT, but the calculated energy of this double-keto structure is $\sim 0.27 \mathrm{eV}$ higher than the minima accessed by single ESIPT and $\sim 0.2 \mathrm{eV}$ above the starting doubleenol minimum. The calculated energy barrier associated with

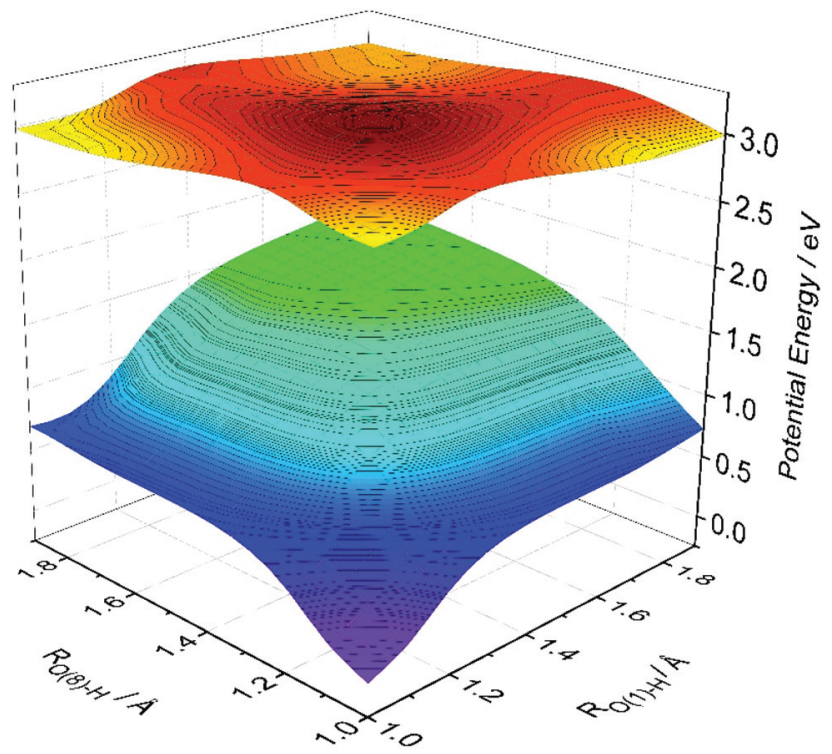

Fig. 4 Two-dimensional potentials for single and double proton transfer in the $S_{0}$ and $S_{1}$ states of HDBO. The PESs were constructed by progressively extending $R_{\mathrm{O}(1)-\mathrm{H}}$ and $R_{\mathrm{O}(8)-\mathrm{H}}$ and, at each chosen geometry, allowing the remaining internal degrees of freedom to relax to the minimum energy structure of the ground state. the concerted double-enol $\rightarrow$ double-keto tautomerism on the $\mathrm{S}_{1}$ PES is $\sim 0.3 \mathrm{eV}$ ( $c f . \sim 1.75 \mathrm{eV}$ on the $\mathrm{S}_{0} \mathrm{PES}$ ). Based on this topography, we predict a dominant role for single ESIPT ( $c f$. double ESIPT) in the photochemistry of HDBO.

As Fig. 3 showed, the unrelaxed PE profiles for the $S_{2}$ and $\mathrm{S}_{3}$ states of both $\mathrm{HBO}$ and HDBO show local minima for both the enol and keto tautomers and can thus also be expected to exhibit CT character upon increasing $R_{\mathrm{O}(1)-\mathrm{H}}$. These unrelaxed potentials all show barriers upon extending $R_{\mathrm{O}(1)-\mathrm{H}}$, beyond which the $\mathrm{S}_{2}$ states (in particular) stabilise and, in both molecules, are predicted to show an energetic preference for the keto-structure. The $\mathrm{T}_{1}$ states of $\mathrm{HBO}$ and HDBO share the same electronic configurations as the respective $S_{1}$ states. The calculated PE profiles for these triplet states also show minima for both the enol and keto tautomers, whereas the profiles for the $\mathrm{T}_{2}$ and $\mathrm{T}_{3}$ states (the electronic configurations of which are analogous to those of the respective $S_{2}$ and $S_{3}$ states) simply increase with increasing $R_{\mathrm{O}(1)-\mathrm{H}}$ - implying no driving force for ESIPT in these states. We note that the $\mathrm{T}_{2}$ and $\mathrm{T}_{3}$ potentials are predicted to cross other singlet and/or triplet potentials, which could provide non-radiative decay pathways for any triplet state population formed by photoexcitation. However, given the absence of heavy atoms in $\mathrm{HBO}$ or HDBO, we suggest that the photochemistry of these molecules is likely to be dominated by nuclear motions on the singlet potentials.

We now focus on the $S_{1}$ state and the likely photochemistry induced by $\mathrm{S}_{1}-\mathrm{S}_{0}$ absorption in these two molecules. Following photoinduced tautomerism, we consider two possible fates for a keto tautomer in its $\mathrm{S}_{1}$ state: fluorescence and internal conversion. Analogy with molecules like 2-(2'-hydroxyphenyl)benzotriazole $^{23}$ and 2-(2'-hydroxyphenyl)benzothiazole ${ }^{33}$ suggests that $E \rightarrow Z$ isomerism around the $\mathrm{C}(3)=\mathrm{C}(9)$ bond (in the case of $\mathrm{HBO},(\mathrm{C}(6)=\mathrm{C}(17)$ in the case of $\mathrm{HDBO}))$ might offer a route from the keto minimum to a minimum energy (ME)CI between the $\mathrm{S}_{1}$ and $\mathrm{S}_{0}$ PESs. Fig. 5, which shows PE profiles for $E \rightarrow Z$

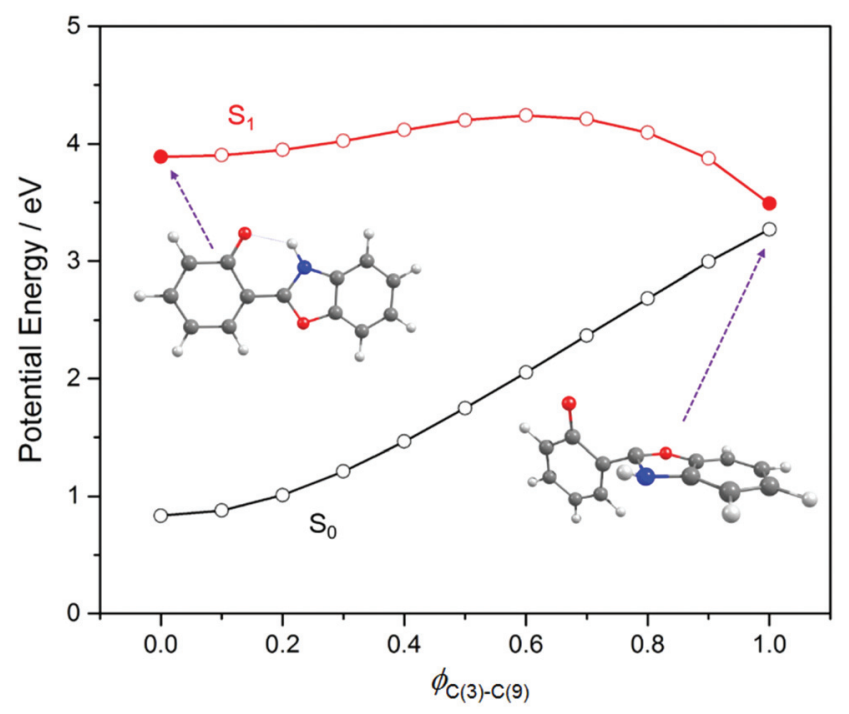

Fig. 5 Calculated PE profiles for $E \rightarrow Z$ isomerism (i.e. by rotating around the $\mathrm{C}(3)=\mathrm{C}(9)$ bond) in the $\mathrm{S}_{0}$ and $\mathrm{S}_{1}$ states of HBO). 
isomerism about $\mathrm{C}(3)=\mathrm{C}(9)$ in $\mathrm{HBO}$ calculated as a function of the $\mathrm{C}(2)-\mathrm{C}(3)-\mathrm{C}(9)-\mathrm{N}(10)$ dihedral angle, $\phi_{\mathrm{C}(3)-\mathrm{C}(9)}$, confirms this expectation. The displayed energy at $\phi_{\mathrm{C}(3)-\mathrm{C}(9)}=0^{\circ}$ is the relaxed $\mathrm{S}_{1}$ energy at $R_{\mathrm{O}(1)-\mathrm{H}}=1.8 \AA$ (see Fig. 3(a)), while the final energy point (at $\phi_{\mathrm{C}(3)-\mathrm{C}(9)}=110^{\circ}$ ) is for the geometry at which the $S_{1} / S_{0}$ energy gap in the initial $S_{1}$ relaxed scan along $\phi_{\mathrm{C}(3)-\mathrm{C}(9)}$ was smallest. The $\mathrm{S}_{1}$ electronic configuration varies across the intermediate geometries spanned by the $S_{1}$ relaxed scan, so the intermediate geometries and associated energies were obtained by constructing a linear interpolation in internal coordinate (LIIC) between $\phi_{\mathrm{C}(3)-\mathrm{C}(9)}=0^{\circ}$ and $110^{\circ}$. The $\sim 0.4 \mathrm{eV}$ barrier in the PE profile returned in this way thus represents an upper limit. Thus the present calculations serve to reinforce previous conclusions regarding the twisted minimum energy geometry of the keto-tautomer of $\mathrm{HBO}$ in its $\mathrm{S}_{1}$ state. $^{42}$ They also suggest that $E \rightarrow Z$ isomerism about the $\mathrm{C}(3)=\mathrm{C}(9)$ bond could be a viable non-radiative decay route by which keto $\mathrm{HBO}\left(\mathrm{S}_{1}\right)$ population (and, by extrapolation, similar populations in $\mathrm{HDBO}$ and the $\mathrm{X}-\mathrm{HDBO}^{\prime}$ complexes) could undergo internal conversion to the $\mathrm{S}_{0}$ state. We will revisit this path later.

Fluorescence is another possible $S_{1}$ population loss mechanism, evidence for which is provided by the reported (albeit weak) emission spectra of the present systems. ${ }^{34}$ Fig. 6 shows $\mathrm{S}_{1}-\mathrm{S}_{0}$ absorption and emission profiles of both $\mathrm{HBO}$ and HDBO calculated using Wigner sampling methods and Newton-X. The respective absorption spectra (shown in blue) are dominated by the parent enol tautomer, while the displayed emission spectra are from both the keto tautomer formed upon single ESIPT (shown in red) and, for completeness, from the respective $\mathrm{S}_{1}$ enol minima (shown in purple).

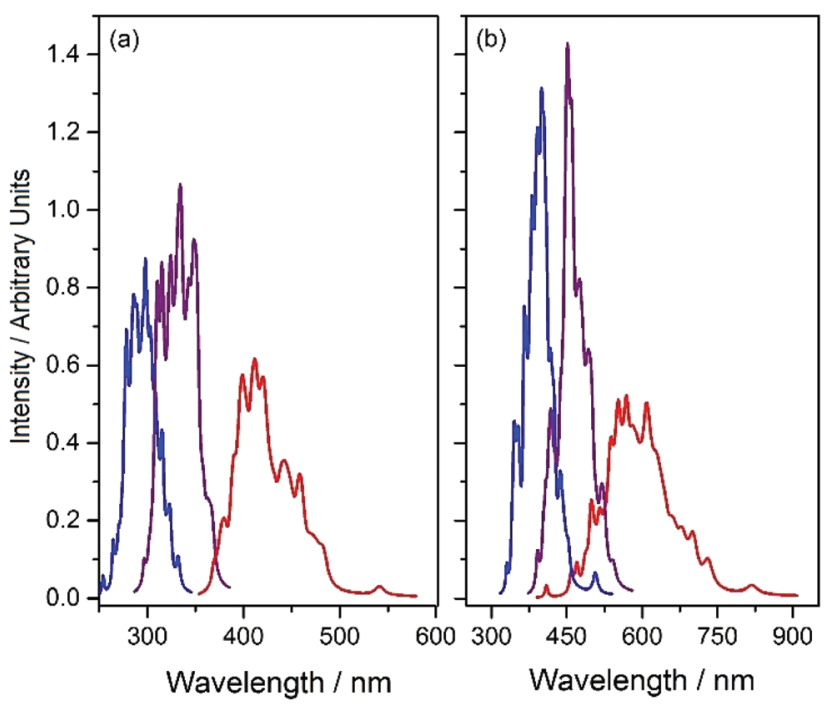

Fig. 6 Calculated gas phase absorption and emission profiles of (a) $\mathrm{HBO}$ and (b) HDBO. The blue trace reflects vertical excitation from the $S_{0}$ enol minimum, while the calculated emissions displayed in red and purple originate from, respectively, the keto and enol minima on the $S_{1}$ PES. For display purposes, the three spectra in each panel are shown with the same peak areas.
These calculations reinforce previous suggestions that single ESIPT is responsible for the large Stokes shifts observed experimentally, ${ }^{34}$ and succeed in reproducing (semi-quantitatively, at least) the magnitudes of these shifts which, as Fig. 6 shows, are predicted to be $\sim 110 \mathrm{~nm}$ in $\mathrm{HBO}$ and $\sim 150 \mathrm{~nm}$ in HDBO.

The present results agree well with previous explorations of the excited state proton transfer of $\mathrm{HBO}$ and HDBO (and substituted analogues) and serve to re-emphasise the necessity for long-range correlation in describing the charge-separated states involved in ESIPT. ${ }^{27,42}$ The extents to which these earlier conclusions, and the available experimental data, are reproduced by the present long-range corrected CAM-B3LYP functional encourages confidence in extrapolating the mechanism identified in $\mathrm{HBO}$ and HDBO to the metal-containing systems.

$\mathbf{X}-\mathbf{H D B O}^{\prime}$ (where $\mathbf{X}=\mathbf{Z n}^{2+}, \mathbf{C d}^{2+}$ and $\mathbf{H g}^{2+}$ ). The calculated PE profiles along the ESIPT coordinate of $\mathrm{Zn}^{2+}-\mathrm{HDBO}^{\prime}$ displayed in Fig. 7 show many similarities with those obtained for $\mathrm{HBO}$ and HDBO. Again, the minimum energy configuration in the $\mathrm{S}_{0}$ state is the enol tautomer, which is predicted to undergo enol $\rightarrow$ keto tautomerism following excitation to the $\mathrm{S}_{1}$ state. The $\mathrm{S}_{1}$ keto tautomer is predicted to be slightly more stable than the enol form (by $\sim 0.04 \mathrm{eV}$ ). The $\mathrm{S}_{1}$ keto minimum is calculated to lie $\sim 2.7 \mathrm{eV}$ above the $\mathrm{S}_{0}$ minimum but, because of the very different topographies of the $S_{1}$ and $S_{0}$ PESs, the $\mathrm{S}_{1}-\mathrm{S}_{0}$ energy gap at the geometry of the $\mathrm{S}_{1}$ keto tautomer is only $\sim 1.7 \mathrm{eV}$ (corresponding to a wavelength of $\sim 720 \mathrm{~nm}$ ). As Fig. 7 shows, the $\mathrm{T}_{1}$ PES shows a similar topography along $R_{\mathrm{O}(1) \mathrm{H}-\mathrm{N}(10)}$ coordinate (consistent with the similar electronic configurations of the $\mathrm{S}_{1}$ and $\mathrm{T}_{1}$ states), hinting at a similar propensity for ESIPT.

Given these topographies, we can now suggest interpretations for the reported $\mathrm{X}-\mathrm{HDBO}^{\prime}$ emission spectra and their

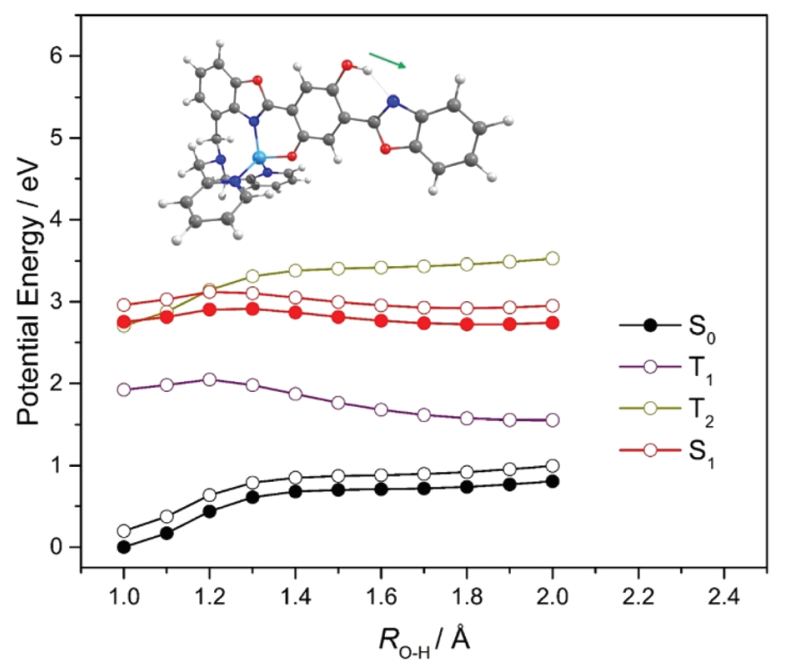

Fig. 7 PE profiles for ground and first few singlet and triplet excited states of $\mathrm{Zn}^{2+}-\mathrm{HDBO}^{\prime}$ along the $R_{\mathrm{O}(1) \mathrm{H}-\mathrm{N}(10)}$ ESIPT coordinate. The black and red filled circles show the relaxed profiles for, respectively, the $S_{0}$ and $S_{1}\left(11 \pi \pi^{*}(C T)\right)$ states. The open black circles show the energy of the $\mathrm{S}_{0}$ state computed at the relaxed $\mathrm{S}_{1}$ state geometries. Other PE profiles shown by colored open circles are for the excited states indicated, calculated at the relaxed $\mathrm{S}_{0}$ state geometries. 

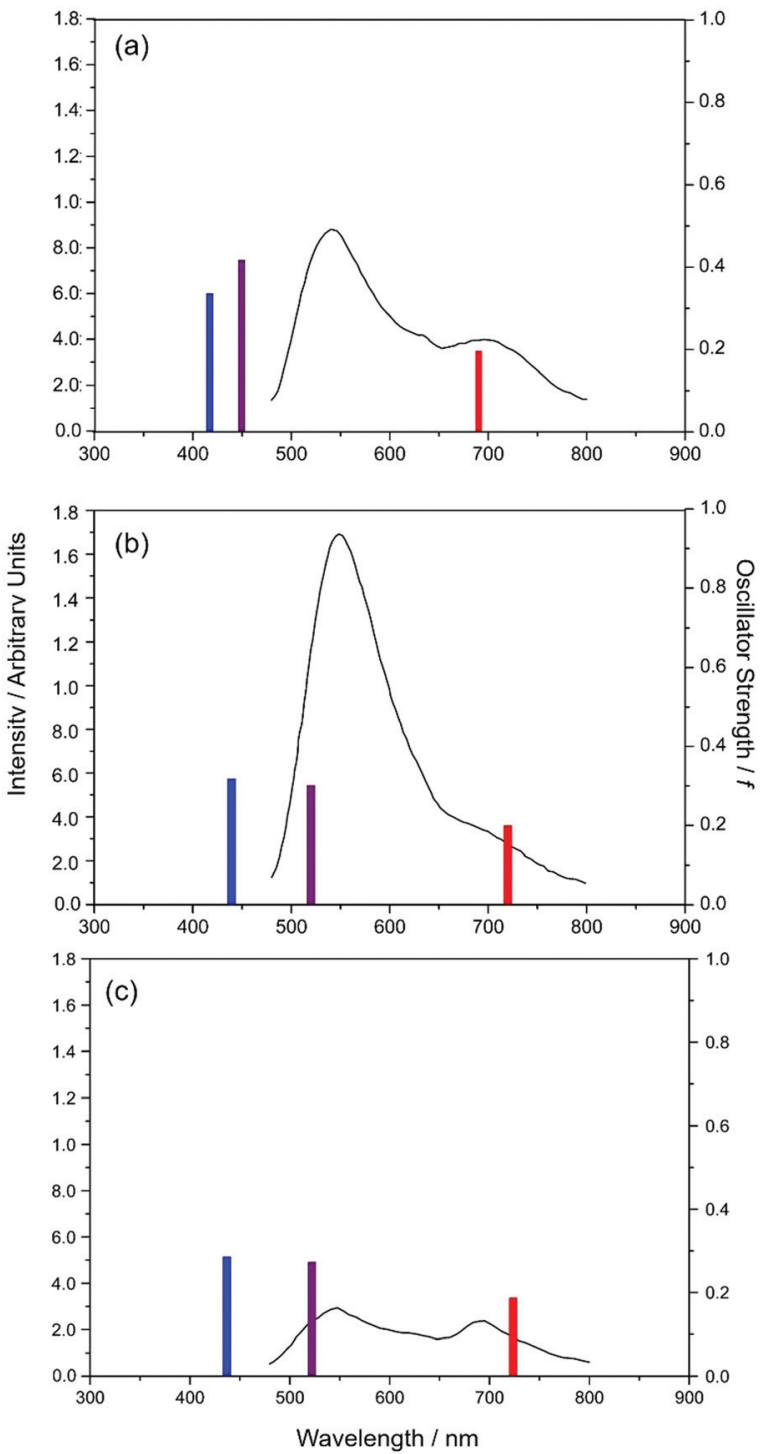

Fig. 8 Comparison between calculated gas phase absorption and emission maxima for (a) $\mathrm{Zn}^{2+}-\mathrm{HDBO}^{\prime}$, (b) $\mathrm{Cd}^{2+}-\mathrm{HDBO}^{\prime}$ and (c) $\mathrm{Hg}^{2+}-\mathrm{HDBO}^{\prime}$ and the experimentally measured emission spectra of these same complexes in aqueous solution (from ref. 31 and shown by the solid black curves). The calculated wavelengths for vertical $S_{1} \leftarrow S_{0}$ absorption from the respective optimised $S_{0}$ geometries are shown by blue sticks, while the peak wavelengths for $S_{1} \rightarrow S_{0}$ emission from the optimised $S_{1}$ enol and keto geometries are shown by, respectively, the purple and red sticks. The different stick heights reflect the respective calculated oscillator strengths (shown on the right hand vertical axis).

low fluorescence quantum yields. ${ }^{34}$ The experimental emission spectra are reproduced in Fig. 8, along with sticks showing the calculated wavelengths of peak $S_{1}-S_{0}$ absorption and of peak emission from both the enol and keto minima on the $\mathrm{S}_{1}$ PES. The present analysis suggests that, as with HBO and HDBO, the longest wavelength emission arises from the keto $\mathrm{S}_{1}$ minimum. This emission shows a substantial bathochromic shift, larger than in the cases of HBO or HDBO, and consistent with experiment. The low fluorescence quantum yield from the $\mathrm{X}-\mathrm{HDBO}^{\prime}$ complexes is plausibly attributed to competition from internal conversion via $E \rightarrow Z$ isomerisation of the keto tautomer, as demonstrated here for the simpler HBO system. Nonetheless, $\mathrm{HDBO}^{\prime}$ satisfies many of the requirements of a sensor for trace metal detection, i.e. the $\mathrm{X}-\mathrm{HDBO}^{\prime}$ complex absorbs at (short) visible wavelengths and fluoresces with a sufficient quantum yield and a large Stokes shift.

As Fig. 8 shows, the experimental spectra display an additional feature at $\sim 540 \mathrm{~nm}$ that has no analogue in the cases of $\mathrm{HBO}$ and $\mathrm{HDBO}$. Xu and Pang noted the very similar excitation profiles for the 540 and $712 \mathrm{~nm}$ emissions from $\mathrm{Zn}^{2+}-\mathrm{HDBO}^{\prime}$ and concluded that both emissions were from the same complex. ${ }^{34}$ Given the PE profiles shown in Fig. 7, we can propose the following explanation for the emissions observed in the solution phase experiments. Vertical excitation projects the $\mathrm{X}-\mathrm{HDBO}^{\prime}$ complex to energies above the $\mathrm{S}_{1}$ minimum. The excited state molecules so formed will evolve under the influence of the $S_{1}$ PES, and start dissipating internal energy by interacting with the surrounding solvent. Some fraction of the $\mathrm{S}_{1}$ state population will undergo ESIPT. This fraction can then make a radiationless transition to the ground state (e.g. by $E \rightarrow$ $Z$ isomerism) or relax into the $\mathrm{S}_{1}$ keto minimum and decay by fluorescing (at $\lambda_{\mathrm{em}} \sim 712 \mathrm{~nm}$ ). Any $\mathrm{S}_{1}$ state population that relaxes into the $\mathrm{S}_{1}$ enol minimum $\left(R_{\mathrm{O}(1)-\mathrm{H}}=1.0 \AA\right)$, however, would likely fluoresce at $\lambda_{\mathrm{em}} \sim 540 \mathrm{~nm}-$ as suggested by Xu and Pang. ${ }^{34}$ Recalling the experimental emission profiles (Fig. 8), it is tempting to suggest that the latter process is (relatively) most important in $\mathrm{Cd}^{2+}-\mathrm{HDBO}^{\prime}$. This could be explained if the energy barrier to enol $\rightarrow$ keto tautomerism on the $\mathrm{S}_{1}$ PES is larger in $\mathrm{Cd}^{2+}-\mathrm{HDBO}^{\prime}$ than in $\mathrm{Zn}^{2+}-\mathrm{HDBO}^{\prime}$. As an alternative, however, the observation could also indicate that, relative to $\mathrm{Zn}^{2+}-\mathrm{HDBO}^{\prime}$, the $\mathrm{S}_{1}$ keto form of $\mathrm{Cd}^{2+}-\mathrm{HDBO}^{\prime}$ has a smaller barrier to $E \rightarrow Z$ isomerism (and thus a lower fluorescence quantum yield for this tautomer).

We note that the present calculations for the isolated (i.e. gas phase) systems cannot allow for the additional complexities that would manifest from considering the bulk aqueous environment. Electronic absorptions to charge-separated states typically show a bathochromic shift in polar solutions (reflecting the stabilisation of the charge-separated state). Notwithstanding, the absorption and emission maxima returned by the present (isolated molecule) calculations are seen to mimic the experimental (aqueous phase) data sufficiently well to enable interpretation of the observed photophysics. Inspecting Fig. 8, it is clear that the peak emission wavelength of the enol-form of $\mathrm{Zn}^{2+}-\mathrm{HDBO}^{\prime}$ is reproduced least well; relative to the other $\mathrm{X}-\mathrm{HDBO}^{\prime}$ species, we predict a notably smaller Stokes shift for $\mathrm{Zn}^{2+}-\mathrm{HDBO}^{\prime}$, implying a smaller change in equilibrium geometry upon $\mathrm{S}_{1} \leftarrow \mathrm{S}_{0}$ excitation in this case. It is unclear at this stage to what extent this is a real effect, or rather a reflection of the different basis sets used for $\mathrm{Zn}^{2+}-\mathrm{HDBO}^{\prime}\left(c f\right.$. $\mathrm{Cd}^{2+}-\mathrm{HDBO}^{\prime}$ and $\mathrm{Hg}^{2+}$ $\left.\mathrm{HDBO}^{\prime}\right)$.

Having offered a rationale for the large and, from the sensing perspective, highly desirable Stokes shifts exhibited by these $\mathrm{X}-\mathrm{HDBO}^{\prime}$ complexes, we now turn attention to potential 

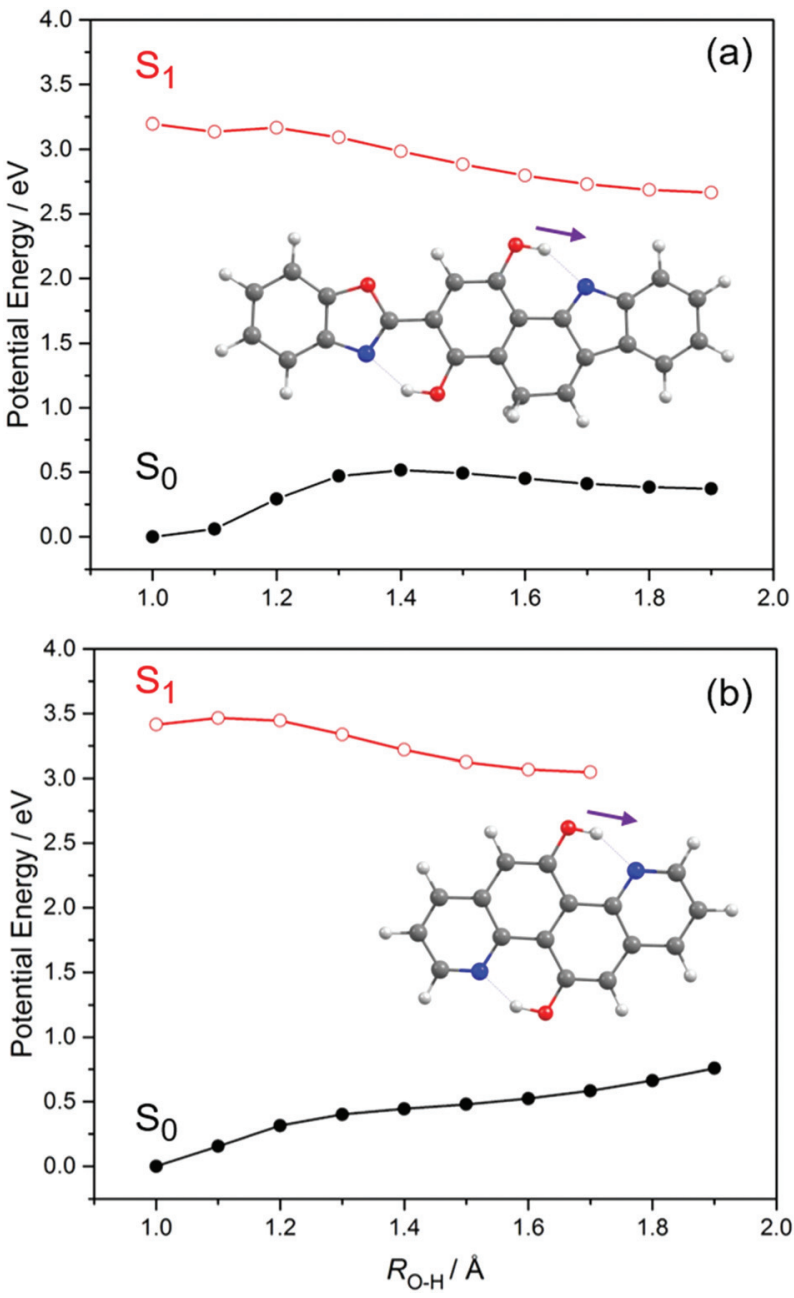

Fig. 9 PE profiles for the $S_{0}$ and $S_{1}$ states of $B C-H B O$ and $Q Q$ along the $R_{\mathrm{O}(1) \mathrm{H}-\mathrm{N}(10)}$ ESIPT coordinate.

modifications of the ligand that might result in a larger fluorescence quantum yield (i.e. minimise the probabilities of competing non-radiative excited state decay processes). Two possible structures are shown in Fig. 9. 3-Benzo[d]oxazol-2-yl$5 H$-benzo[a]carbazole-1,4-diol (henceforth BC-HBO, shown in Fig. 9(a)), is an analogue of HDBO in which $\mathrm{O}(13)$ is replaced by a $\mathrm{C}$ atom (henceforth labelled $\mathrm{C}(18)$ ) and a two-carbon bridge links $\mathrm{C}(18)$ to $\mathrm{C}(4)$. As Fig. 9(a) shows, BC-HBO is predicted to absorb at similar wavelengths to $\mathrm{HDBO}$, to undergo ESIPT to the keto-tautomer upon $\mathrm{S}_{1} \leftarrow \mathrm{S}_{0}$ excitation, and to show substantially red-shifted $\mathrm{S}_{1} \rightarrow \mathrm{S}_{0}$ emission. Crucially, however, the two-carbon bridge linking $\mathrm{C}(18)$ to $\mathrm{C}(4)$ not only holds all atoms in a common plane, but should mitigate against any non-radiative population loss via rotation about $\phi_{\mathrm{C}(3)-\mathrm{C}(9)}$ in the keto-conformer. Fig. 9(b) depicts another analogue of HDBO, quinolino[8,7-h]quinolone-5-11-diol (henceforth QQ), which is calculated to display similar tendencies for ESIPT, red-shifted $\mathrm{S}_{1} \rightarrow \mathrm{S}_{0}$ emission and, again, the additional structural rigidity to preclude excited state $E \rightarrow Z$ isomerism as a rival to fluorescence decay. Analogy with
HDBO and, particularly, the deprotonated variant of $\mathrm{HDBO}^{\prime}$, suggests that suitably derivitized variants of either of these ligands could constitute improved ESIPT-based sensors for trace metal detection.

\section{Conclusions}

The present computational study reveals mechanistic details of a prototypical system that can function as a fluorescent sensor for trace metal cations. We have scanned PE profiles along selected coordinates for the ground and first few excited states for the target metal-chelated complexes (X-HDBO', with $\mathrm{X}=\mathrm{Zn}^{2+}, \mathrm{Cd}^{2+}$ and $\mathrm{Hg}^{2+}$ ) and for simpler molecular building blocks like $\mathrm{HBO}$ and HDBO from which these complexes derive, and shown that all are likely to undergo ESIPT following $S_{1} \leftarrow S_{0}$ photoexcitation. The initial excitation in all of these cases involves a $\pi^{*} \leftarrow \pi$ transition, that induces charge separation in the $S_{1}$ state and drives a subsequent proton transfer which, in these molecules, is synonymous with enol $\rightarrow$ keto tautomerism. In each case, the resulting keto-tautomer constitutes a local minimum on the $\mathrm{S}_{1}$ PES, and is identified as the carrier of Stokes shifted fluorescent emission - consistent with recent experimental studies ${ }^{34}$ that reveal measurable fluorescence quantum yield from this tautomer. The present study succeeds in reproducing the relative energies of the observed $\mathrm{S}_{1} \leftarrow \mathrm{S}_{0}($ enol $)$ absorptions across the series $\mathrm{HBO}$, HDBO, protonated and deprotonated $\mathrm{HDBO}^{\prime}$ and the X-HDBO' complexes themselves, and of their respective $\mathrm{S}_{1}($ keto $) \rightarrow \mathrm{S}_{0}$ emissions. We also suggest $\mathrm{S}_{1}($ enol $) \rightarrow \mathrm{S}_{0}$ fluorescence as an explanation for hitherto unassigned shorter wavelength emission from the $\mathrm{X}-\mathrm{HDBO}^{\prime}$ complexes. Derivatives of $\mathrm{HDBO}^{\prime}$ are also proposed that are predicted to retain attractive features like (short wavelength) visible absorption and heavily Stokes shifted emission but, additionally, offer higher fluorescence quantum yields (i.e. enhanced metal sensing capability) by precluding possible non-radiative decay via $E \rightarrow Z$ isomerism from the $\mathrm{S}_{1}$ keto minimum to the $\mathrm{S}_{0}$ PES.

\section{Acknowledgements}

The authors are grateful to the Engineering and Physical Sciences Research Council via Programme grant EP/L005913/1. $\mathrm{BM}$ and TNVK are grateful to TUM for the award of postdoctoral research fellowships. The underlying data upon which this manuscript is based has been stored in the University of Bristol research data repository and can be access via the following link: DOI: 10.5523/bris.836bu3nhnbzg1pszfc2xowr44.

\section{Notes and references}

1 L. Basabe-Desmonts, D. N. Reinhoudt and M. CregoCalama, Chem. Soc. Rev., 2007, 36, 993-1017.

2 R. N. Dsouza, U. Pischel and W. M. Nau, Chem. Rev., 2011, 111, 7941-7980. 
3 A. B. Chinen, C. M. Guan, J. R. Ferrer, S. N. Barnaby, T. J. Merkel and C. A. Mirkin, Chem. Rev., 2015, 115, 1053010574.

4 K. P. Carter, A. M. Young and A. E. Palmer, Chem. Rev., 2014, 114, 4564-4601.

5 E. A. Lemke and C. Schultz, Nat. Chem. Biol., 2011, 7, 480483.

6 K. J. Wallace, Supramol. Chem., 2009, 21, 89-102.

7 A. P. de Silva, H. Q. Nimal Gunaratne and K. R. A. Samankumara Sandanayake, Tetrahedron Lett., 1990, 31, 5193-5196.

8 M. S. Koay, B. M. G. Janssen and M. Merkx, Dalton Trans., 2013, 42, 3230-3232.

9 S. Bakthavatsalam, A. Sarkar, A. Rakshit, S. Jain, A. Kumar and A. Datta, Chem. Commun., 2015, 51, 2605-2608.

10 S. Arimori, G. A. Consiglio, M. D. Phillips and T. D. James, Tetrahedron Lett., 2003, 44, 4789-4792.

11 K. K. Sadhu, S. Sen and P. K. Bharadwaj, Dalton Trans., 2011, 40, 726-734.

12 D. C. Magri, G. J. Brown, G. D. McClean and A. P. de Silva, J. Am. Chem. Soc., 2006, 128, 4950-4951.

13 J. M. Tarascon, G. W. Hull and F. J. DiSalvo, Mater. Res. Bull., 1984, 19, 915-924.

14 V. Daniel, R. Maja, J. Adolf, M. Aleš, U. Polona, P. Maja, J. Boštjan, M. Anton, N. Barbara, P. Stane, V. Peter, J. C. Coleman and M. Dragan, Nanotechnology, 2004, 15, 635.

15 D. Mihailovic, Prog. Mater. Sci., 2009, 54, 309-350.

16 X. Yin, S. A. Warren, Y.-T. Pan, K.-C. Tsao, D. L. Gray, J. Bertke and H. Yang, Angew. Chem., Int. Ed., 2014, 53, 14087-14091.

17 E. Merino and M. Ribagorda, Beilstein J. Org. Chem., 2012, 8, 1071-1090.

18 P. Hamm, S. M. Ohline and W. Zinth, J. Chem. Phys., 1997, 106, 519-529.

19 I. K. Lednev, T. Q. Ye, P. Matousek, M. Towrie, P. Foggi, F. V. R. Neuwahl, S. Umapathy, R. E. Hester and J. N. Moore, Chem. Phys. Lett., 1998, 290, 68-74.

20 W. Domcke, D. R. Yarkony and H. Koppel, Conical Intersections: Electronic Structure, Dynamics and Spectroscopy, World Scientific, Singapore, 2004.

21 S. Hammes-Schiffer, J. Am. Chem. Soc., 2015, 137, 88608871.

22 A. L. Sobolewski and W. Domcke, Phys. Chem. Chem. Phys., 2006, 8, 3410-3417.
23 A. L. Sobolewski, W. Domcke and C. Hättig, J. Phys. Chem. A, 2006, 110, 6301-6306.

24 M. F. Rode and A. L. Sobolewski, Chem. Phys., 2012, 409, 41-48.

25 T. N. V. Karsili, B. Marchetti, M. N. R. Ashfold and W. Domcke, J. Phys. Chem. A, 2014, 118, 11999-12010.

26 Q. L. Nguyen, V. A. Spata and S. Matsika, Phys. Chem. Chem. Phys., 2016, 18, 20189-20198.

27 C. Azarias, S. Budzak, A. D. Laurent, G. Ulrich and D. Jacquemin, Chem. Sci., 2016, 7, 3763-3774.

28 H. Wang, H. Zhang, O. K. Abou-Zied, C. Yu, F. E. Romesberg and M. Glasbeek, Chem. Phys. Lett., 2003, 367, 599-608.

29 T. Arthen-Engeland, T. Bultmann, N. P. Ernsting, M. A. Rodriguez and W. Thiel, Chem. Phys., 1992, 163, 4353.

30 O. K. Abou-Zied, R. Jimenez, E. H. Z. Thompson, D. P. Millar and F. E. Romesberg, J. Phys. Chem. A, 2002, 106, 3665-3672.

31 S. Lochbrunner, K. Stock and E. Riedle, J. Mol. Struct., 2004, 700, 13-18.

32 R. Daengngern and N. Kungwan, Chem. Phys. Lett., 2014, 609, 147-154.

33 D. T. Mancini, K. Sen, M. Barbatti, W. Thiel and T. C. Ramalho, ChemPhysChem, 2015, 16, 3444.

34 Y. Xu and Y. Pang, Chem. Commun., 2010, 46, 4070-4072.

35 M. J. Frisch, G. W. Trucks, H. B. Schlegel, G. E. Scuseria, M. A. Robb, J. R. Cheeseman, G. Scalmani, V. Barone, B. Mennuchi, G. Petersson, et al., Gaussian 09, 2010, revision B.01, Gaussian Inc., Wallingford, CT.

36 T. Yanai, D. P. Tew and N. C. Handy, Chem. Phys. Lett., 2004, 393, 51-57.

37 W. J. Hehre, R. F. Stewart and J. A. Pople, J. Chem. Phys., 1969, 51, 2657.

38 W. R. Wadt and P. J. Hay, J. Chem. Phys., 1985, 82, 284-298.

39 M. Barbatti, G. Granucci, M. Persico, M. Ruckenbauer, M. Vazdar, M. Eckert-Maksić and H. Lischka, J. Photochem. Photobiol., A, 2007, 190, 228-240.

40 M. Barbatti, M. Ruckenbauer, F. Plasser, J. Pittner, G. Granucci, M. Persico and H. Lischka, Wiley Interdiscip. Rev.: Comput. Mol. Sci., 2014, 4, 26-33.

41 M. Barbatti, A. J. A. Aquino and H. Lischka, Phys. Chem. Chem. Phys., 2010, 12, 4959-4967.

42 Y. Syetov, J. Fluoresc., 2013, 23, 689-696. 